\title{
Microwave Background Anisotropies Due to the Kinematic Sunyaev-Zeldovich Effect of the Lya Forest
}

\section{Citation}

Loeb, Abraham. 1996. "Microwave Background Anisotropies Due to the Kinematic SunyaevZeldovich Effect of the Lya Forest." The Astrophysical Journal 471 (1): L1-4. https:// doi.org/10.1086/310317.

\section{Permanent link}

http://nrs.harvard.edu/urn-3:HUL.InstRepos:41393369

\section{Terms of Use}

This article was downloaded from Harvard University's DASH repository, and is made available under the terms and conditions applicable to Other Posted Material, as set forth at http:// nrs.harvard.edu/urn-3:HUL.InstRepos:dash.current.terms-of-use\#LAA

\section{Share Your Story}

The Harvard community has made this article openly available. Please share how this access benefits you. Submit a story.

Accessibility 
The Astrophysical Journal, 471:L1-L4, 1996 November 1

(c) 1996. The American Astronomical Society. All rights reserved. Printed in U.S.A.

\title{
MICROWAVE BACKGROUND ANISOTROPIES DUE TO THE KINEMATIC SUNYAEV-ZELDOVICH EFFECT OF THE Ly $\alpha$ FOREST
}

\author{
ABRAHAm Loeb \\ Astronomy Department, Harvard University, 60 Garden Street, Cambridge, MA 02138 \\ Received 1996 April 11; accepted 1996 August 15
}

\begin{abstract}
The Ly $\alpha$ absorption systems observed in the spectra of QSOs are likely to possess bulk peculiar velocities. The free electrons in these systems scatter the microwave background and distort its spectrum through the kinematic Sunyaev-Zeldovich effect. I calculate the temperature fluctuations of the microwave sky due to variations in the number of $\operatorname{Ly} \alpha$ systems along different lines of sight throughout the universe. The known population of absorbers out to $z \approx 5$ introduces anisotropies on angular scales $\lesssim 1^{\prime}$ with an rms amplitude of order $\Delta T / T \approx$ $10^{-6}\left(\Omega_{\mathrm{Ly} \alpha} / 0.05\right)\left\langle v_{400}^{2}\right\rangle^{1 / 2}$, where $\Omega_{\mathrm{Ly} \alpha}$ is the cosmological density parameter of ionized gas in Ly $\alpha$ absorption systems, and $\left\langle v_{400}^{2}\right\rangle^{1 / 2}$ is the rms line-of-sight peculiar velocity of these systems at $z \sim 3$ in units of $400 \mathrm{~km} \mathrm{~s}$. Detection of this signal will provide valuable information about the cosmic velocity field and the gas content of Ly $\alpha$ absorption systems at high redshifts.
\end{abstract}

Subject headings: cosmic microwave background — cosmology: theory — quasars: absorption lines

\section{INTRODUCTION}

Sunyaev \& Zeldovich showed that Compton scattering off free electrons would distort the spectrum of the cosmic background radiation (CBR) either if the electrons have a different temperature than the radiation or if they possess a bulk velocity relative to the cosmic frame (see Sunyaev \& Zeldovich 1980 for a review). By now, the thermal effect has been seen in many clusters of galaxies (Birkinshaw 1993; Rephaeli 1995), and observations to measure the kinematic effect are underway (see discussion in Haehnelt \& Tegmark 1995). The integrated level of distortion introduced to the CBR spectrum by intergalactic gas is currently unknown, although it may be considerable in popular cold dark matter cosmologies (Colafrancesco et al. 1994, 1995; Persi et al. 1995). The perfect blackbody spectrum detected by the $C O B E$ satellite sets a tight limit on the total Comptonization parameter of the universe, $y \leq 1.5 \times 10^{-5}$ (Fixsen et al. 1996). Although this limit can be used to rule out exotic cosmological scenarios (Levin, Freese, \& Spergel 1992; Wright et al. 1994), it still allows for a significant contribution to the observed CBR anisotropies by the Sunyaev-Zeldovich (SZ) effect. As future satellite experiments are currently being designed with the hope of mapping the microwave sky on angular scales $\gtrsim 10^{\prime}$ (Janssen et al. 1996), it is important to characterize the unavoidable contribution from intergalactic scattering to the expected anisotropies.

The intergalactic medium at redshifts $z \lesssim 5$ is highly ionized and inhomogeneous. Direct evidence for condensations of gas comes from the absorption lines observed in QSO spectra (see reviews in Blades, Turnshek, \& Norman 1988). Close QSO pairs and multiple images of gravitationally lensed QSOs indicate that the transverse extent of these absorption systems is $\ell_{\perp} \sim 0.1-1 \mathrm{Mpc}$ (Bechtold et al. 1994; Dinshaw et al. 1994, 1995; Fang et al. 1995; Smette et al. 1995). The neutral hydrogen density in these systems is thought to be controlled through photoionization equilibrium by an ionizing radiation background. The existence of this background radiation has been established by the "proximity effect," with the background flux estimated from the size of the ionization region surrounding bright QSOs (e.g., Bajtlik, Duncan, \& Ostriker 1988; Lu, Wolfe, \& Turnshek 1991; Bechtold 1994). Hydrodynamic simulations of popular cosmological models tend to recover the essential properties of the observed absorption systems and imply that these systems are a generic consequence of early structure formation in the universe (Cen et al. 1994; Zhang, Anninos, \& Norman 1995; Hernquist et al. 1996; Miralda-Escudé et al. 1996).

Like other objects in the universe, the $\operatorname{Ly} \alpha$ absorption systems are expected to develop bulk peculiar velocities in response to the gravitational field in their vicinity. Thus, as the free electrons in an absorption system scatter the microwave background, they would distort its spectrum through the kinematic Sunyaev-Zeldovich effect. For a single absorption system with a line-of-sight peculiar velocity of $|v| \gtrsim 100 \mathrm{~km} \mathrm{~s}^{-1}$ and a characteristic temperature in the range $T \sim 10^{4-5} \mathrm{~K}$, the kinematic SZ effect is much larger than the thermal SZ effect since $|v / c| \gtrsim 10\left(2 \mathrm{kT} / \mathrm{m}_{e} c^{2}\right)$, where $m_{e}$ is the electron rest mass. This is the opposite situation from that in clusters of galaxies. The Rayleigh-Jeans distortion is then $(\Delta T / T) \approx-\tau v / c$, where $\tau$ is the optical depth of the $\operatorname{Ly} \alpha$ system to Thomson scattering. The contributions from positive and negative velocities of individual systems would tend to cancel out on average, and the integrated CBR spectrum over the whole sky would therefore show little evidence for this distortion. However, in small regions of the sky, of order the angular scale occupied by a single Ly $\alpha$ absorption system $\left(\lesssim 1^{\prime}\right)$, there could be large rms amplitude of fluctuations due to incomplete cancellations between a small number of absorption systems. In this Letter, I calculate these fluctuations and show that their detection could provide important new information about Ly $\alpha$ absorption systems at high redshifts.

\section{CALCULATION}

Our discussion is based on the assumption that the intergalactic gas at high redshifts is in photoionization equilibrium 
with a UV radiation background. The time required to establish photoionization equilibrium, $\sim 10^{4} J_{21}^{-1} \mathrm{yr}$, is much shorter than the Hubble time, for the Lyman-limit intensity inferred from the proximity effect (Bechtold 1994), $J_{v}=$ $J_{21} 10^{-21}$ ergs $\mathrm{cm}^{-2} \mathrm{~s}^{-1} \mathrm{~Hz}^{-1} \mathrm{sr}^{-1}$. We focus our attention on systems with column densities below the Lyman limit, $N \leq$ $N_{\max }=10^{17} \mathrm{~cm}^{-2}$, because thicker systems are opaque to photoionizing photons and are therefore composed primarily of neutral hydrogen that does not interact with the microwave background.

Let us first find the rms amplitude of fluctuations in the optical depth of the universe due to the clumpiness of the intergalactic gas. Most of this gas is known to be ionized, and its neutral hydrogen component serves as a tracer of the fluctuations in the electron density. At photoionization equilibrium, the clumpiness in the electron density is directly proportional to the local neutral hydrogen density, $n_{e}^{2} \propto n_{\mathrm{H}}$. Thus, overdense regions show up as absorption lines in QSO spectra, and their statistical properties can be inferred from observations. We define $n_{e}$ to be the mean electron density within an absorption system of thickness $\ell$ and $\mathrm{H}$ I column density $N$. At photoionization equilibrium,

$$
n_{e}^{2} \ell=\eta N \text {, }
$$

where $\eta \approx 30 J_{21}\left(T / 5 \times 10^{4} \mathrm{~K}\right)^{0.7} \mathrm{~cm}^{-3}$ (Spitzer 1978, p. 107; Peebles 1993, pp. 556, 558). For the redshift interval of interest, $2 \lesssim z \lesssim 5$, we assume that $J_{21}$ and therefore $\eta$ are constant in time (see discussions in Bajtlik et al. 1988 and Bechtold 1994). The optical depth of an absorption system to Thomson scattering can then be obtained from equation (1),

$$
\tau(N)=\sigma_{\mathrm{T}} n_{e} \bar{\ell}=\sigma_{\mathrm{T}}(\eta N \bar{\ell})^{1 / 2},
$$

where $\sigma_{\mathrm{T}}$ is the Thomson cross section, and $\bar{\ell}(N)$ is the ensemble-averaged thickness of all absorption systems with column density $N$. For simplicity, we parameterize $\bar{\ell}=B N^{-\alpha}$. While low column density systems with $N \sim 10^{14} \mathrm{~cm}^{-2}$ may have a line-of-sight thickness $\bar{\ell} \lesssim 10^{2} \mathrm{kpc}$ (Rauch \& Haehnelt 1995; Bechtold et al. 1994; Dinshaw et al. 1994, 1995; Fang et al. 1995; Smette et al. 1995), damped Ly $\alpha$ absorbers with $N \sim 10^{21} \mathrm{~cm}^{-2}$ are probably protogalactic disks with a characteristic thickness $\bar{\ell} \sim 10^{0-1} \mathrm{kpc}$. This modest variation in thickness over many decades in column density implies a relatively small value of $\alpha \sim 0.2$. As shown later, the value of $B$ does not enter into our final result.

The probability of intersecting a $\operatorname{Ly} \alpha$ absorption system with an $\mathrm{H}$ I column density between $N$ and $N+d N$ in a redshift interval $d z$ around $z \sim 3$ is well fitted by a power law (Carswell et al. 1984; Tytler 1987),

$$
\left(\frac{d P}{d N d z}\right)_{z=3}=A N^{-\beta},
$$

where $A=10^{9.1 \pm 0.3} \mathrm{~cm}^{2 \beta+2}$ and $\beta=1.51 \pm 0.02$ (Sargent, Steidel, \& Boksenberg 1989). Most of the fluctuations in the optical depth of the universe are contributed by systems close to the Lyman limit. For these systems, we extrapolate equation (3) to other redshifts with the scaling,

$$
\frac{d P}{d N d z} \propto(1+z)^{\gamma},
$$

where $\gamma=1.5 \pm 0.4$ (Stengler-Larrea et al. 1995; see also Storrie-Lombardi et al. 1994). Since the systems of interest are separated by large redshift intervals along a given line of sight (e.g., there is typically one Lyman-limit system per unit redshift), we can safely ignore any correlations between them (see also Sargent et al. 1980; Webb \& Barcons 1991) and assume that they are randomly distributed in redshift and column density according to the probability distribution in equations (3) and (4).

We can now calculate the rms level of fluctuations in the optical depth out to $z=5$ due to the finite number of absorption systems along random lines of sight. In each infinitesimal $(\Delta N, \Delta z)$ bin, there is either one or no $\operatorname{Ly} \alpha$ absorption system. The probability of having a system with an optical depth $\tau(N)$ is $p=(d P / d N d z) \Delta N \Delta z \ll 1$. Therefore, the variance of $\tau$ in each bin is $p \tau^{2}-(p \tau)^{2} \approx p \tau^{2}$. The integrated variance is then obtained by summing the independent contributions of all infinitesimal bins,

$$
\begin{aligned}
\left\langle\Delta \tau^{2}\right\rangle & =\int_{0}^{5} d z \int_{N_{\min }}^{N_{\max }} d N \frac{d P}{d N d z} \tau^{2} \\
& =\left[\frac{4.4 \eta A B \sigma_{\mathrm{T}}^{2}}{2-\beta-\alpha}\right]\left(N_{\max }^{2-\beta-\alpha}-N_{\min }^{2-\beta-\alpha}\right),
\end{aligned}
$$

where the second equality follows from equations (2)-(4) with $\gamma=1.5$. As expected, the total rms amplitude of fluctuations grows in proportion to the square root of the number of $\operatorname{Ly} \alpha$ systems, namely, $\left\langle\Delta \tau^{2}\right\rangle^{1 / 2} \propto A^{1 / 2}$. The constant coefficient in square brackets depends on a variety of uncertain parameters, such as $J_{21}, T$, or $B$. We therefore prefer to trade these parameters in favor of a single quantity, the density parameter of ionized gas, whose value can be related to big bang nucleosynthesis. We achieve this conversion by calculating the derivative of the mean optical depth of the universe from equations (2) and (4),

$$
\begin{aligned}
\left(\frac{d\langle\tau\rangle}{d z}\right)_{z=3}= & \int_{N_{\min }}^{N_{\max }} d N\left(\frac{d P}{d N d z}\right)_{z=3} \tau(N) \\
= & {\left[\frac{A(\eta B)^{1 / 2} \sigma_{\mathrm{T}}}{3 / 2-\beta-\alpha / 2}\right] } \\
& \times\left(N_{\max }^{3 / 2-\beta-\alpha / 2}-N_{\min }^{3 / 2-\beta-\alpha / 2}\right) .
\end{aligned}
$$

Since the mean optical depth is invariant to clumpiness for an optically thin medium, we can also express $d\langle\tau\rangle / d z$ in terms of the density parameter of ionized gas in absorption systems below the Lyman limit, $\Omega_{\mathrm{Ly} \alpha}$,

$$
\begin{aligned}
\left(\frac{d\langle\tau\rangle}{d z}\right)_{z=3} & =-\left(\left\langle n_{e}\right\rangle \sigma_{\mathrm{T}} \frac{c d t}{d z}\right)_{z=3} \\
& =5.5 \times 10^{-3}\left[\frac{F\left(\Omega, \Omega_{\Lambda}, 3\right)}{F(0.3,0.7,3)}\right]\left(\frac{\Omega_{\mathrm{Ly} \alpha} h_{50}}{0.05}\right),
\end{aligned}
$$

where $h_{50}$ is the Hubble constant in units of $50 \mathrm{~km} \mathrm{~s}^{-1} \mathrm{Mpc}^{-1}, \Omega$ and $\Omega_{\Lambda}$ are the density parameters of matter and the cosmological constant, respectively, and $F\left(\Omega, \Omega_{\Lambda}, z\right) \equiv(1+z)^{2} /\left[\Omega(1+z)^{3}+\left(1-\Omega-\Omega_{\Lambda}\right)\right.$ $\left.(1+z)^{2}+\Omega_{\Lambda}\right]^{1 / 2}$.

The density of baryons predicted by big bang nucleosynthesis could be in the form of either ionized gas, neutral gas, or compact objects such as stars. The neutral hydrogen can be weighted by the frequency of absorption systems in the spectra 
of QSOs; this yields $\Omega_{\mathrm{H}_{\mathrm{I}}} \approx(1-4) \times 10^{-3}$ at a redshift $z \sim 3$, a value that is comparable to the mass density of luminous stars in the local universe (Lanzetta, Wolfe, \& Turnshek 1995). The metallicity of the densest absorption systems, which are thought to be the progenitors of present-day disk galaxies (the so-called damped Ly $\alpha$ systems), is only $1 / 10$ of the solar value (Lanzetta et al. 1995; Pettini, Lipman, \& Hunstead 1995; Sembach et al. 1995), implying that star formation is only at its infancy at redshifts $z \sim 2-5$. If we therefore assume that the stellar mass fraction is small, with a density parameter $\Omega_{*} \ll$ $\Omega_{\mathrm{H}}$, then most of the baryons in the universe may be in the form of ionized gas at $z \sim 2-5$. Based on the baryonic density parameter predicted by standard big bang nucleosynthesis (Walker et al. 1991), $\Omega_{b} \approx 0.05 h_{50}^{-2}$, it is then plausible to substitute $\Omega_{\mathrm{Ly} \alpha} \sim 0.05$ in equation (7). Indeed, hydrodynamic simulations indicate that more than $85 \%$ of the baryons reside in absorption systems below the Lyman limit (see Fig. 23 in Miralda-Escudé et al. 1996).

By multiplying the square root of equation (5) with the ratio of equations (7) and (6), we get

$$
\begin{aligned}
\left\langle\Delta \tau^{2}\right\rangle^{1 / 2}= & 1.2 \times 10^{-2}\left[\frac{F\left(\Omega, \Omega_{\Lambda}, 3\right)}{F(0.3,0.7,3)}\right] \\
& \times\left(\frac{\Omega_{\mathrm{Ly} \alpha} h_{50}}{0.05}\right) \frac{(-3 / 2+\beta+\alpha / 2)}{[A(2-\beta-\alpha)]^{1 / 2}} \\
& \times \frac{\left(N_{\max }^{(2-\beta-\alpha)}-N_{\min }^{(2-\beta-\alpha)}\right)^{1 / 2}}{\left(N_{\min }^{(3 / 2-\beta-\alpha / 2)}-N_{\max }^{(3 / 2-\beta-\alpha / 2)}\right)} .
\end{aligned}
$$

For $A=10^{9.1} \mathrm{~cm}^{5}, \quad \beta=1.5, \Omega_{\mathrm{Ly} \alpha} h_{50}=0.05, \Omega=0.3$, $\Omega_{\Lambda}=0.7, \alpha=0.2, N_{\max }=10^{17} \mathrm{~cm}^{-2}$, and $N_{\min }=10^{13} \mathrm{~cm}^{-2}$, we get $\left\langle\Delta \tau^{2}\right\rangle^{1 / 2}=7 \times 10^{-4}$. This corresponds to an rms amplitude of fluctuations of order $3 \%$ in the mean optical depth of the universe out to $z=5$. This result has a weak dependence on uncertainties in the values of most of the above parameters (to a first approximation, $\left\langle\Delta \tau^{2}\right\rangle^{1 / 2} \propto N_{\min }^{0.1} N_{\max }^{0.15}$ ).

We finally wish to calculate the resulting fluctuations in the microwave background temperature due to the kinematic Sunyaev-Zeldovich effect of the Ly $\alpha$ forest. For this purpose, we assume that the optical depth $\tau$ and the peculiar velocity $v$ of an absorption system are uncorrelated and then repeat the calculation that led to equation (5). Note that we define $v$ to be the net bulk velocity of an absorption system, and so we average over internal velocities within the system. The resulting rms amplitude of fluctuations in the microwave temperature is

$$
\begin{aligned}
\left(\frac{\Delta T}{T}\right) & =\left\langle\Delta \tau^{2}\right\rangle^{1 / 2}\left\langle(v / c)^{2}\right\rangle^{1 / 2} \\
& \approx 10^{-6}\left(\frac{\Omega_{\mathrm{Ly} \alpha}}{0.05}\right)\left\langle\left(\frac{v}{400 \mathrm{~km} \mathrm{~s}^{-1}}\right)^{2}\right\rangle^{1 / 2},
\end{aligned}
$$

where $\left\langle v^{2}\right\rangle$ is the dispersion in the line-of-sight peculiar velocities of absorption systems, and the second equality was obtained for the set of parameter values chosen after equation (8). The dispersion $\left\langle v^{2}\right\rangle$ is averaged over redshift with a weight factor of $(1+z)^{\gamma}$. The anisotropy signal expressed in equation (9) varies on the angular size of a Ly $\alpha$ absorption system at $z \sim 5, \theta_{\mathrm{Ly} \alpha} \sim 1^{\prime}\left(\ell_{\perp} / 500 h_{50}^{-1} \mathrm{kpc}\right)$, and declines on larger scales roughly as $\propto\left(\theta / \theta_{\mathrm{Ly} \alpha}\right)^{-1}$.

In the local universe, the peculiar velocity field of galaxies shows a characteristic one-dimensional dispersion of order $\sim 500 \mathrm{~km} \mathrm{~s}^{-1}$ (Strauss, Cen, \& Ostriker 1993) and a coherence length of order a few tens of megaparsecs (Górski et al. 1989; Strauss \& Willick 1995). Both linear theory [yielding $v \propto$ $(1+z)^{-0.5}$ for $\Omega=1$ and a more moderate decline for $\Omega<1$ ] and nonlinear dynamics predict that the velocity dispersion would scale down only by a modest factor of order 2 for $z \sim 3$. It is therefore plausible to consider $\left\langle v^{2}\right\rangle^{1 / 2} \sim 300 \mathrm{~km} \mathrm{~s}^{-1}$ in equation (9). The redshift evolution of the Ly $\alpha$ absorber population conspires to almost cancel out the expected decline in the rms peculiar velocity with redshift; for $\Omega=1$, equations (5)-(7) yield $\left\langle\Delta \tau^{2}\right\rangle^{1 / 2} \propto(1+z)^{1-\gamma / 2}=$ $(1+z)^{0.25}$. One should remember, however, that, in a difference from galaxies, pressure forces can influence the peculiar velocity field of Ly $\alpha$ absorption systems, and so naive scalings based on the theory of collisionless gravitational instability may not provide accurate predictions for these systems. Since the SZ fluctuations calculated above are dominated by rare high column density systems that are separated by a path length much longer than the coherence length of the peculiar velocity field, we are justified in ignoring any velocity correlations between neighboring systems on angular scales $\sim \theta_{\text {Ly } \alpha}$.

\section{DISCUSSION}

Equation (9) summarizes the expected temperature fluctuations in the microwave sky due to the kinematic SunyaevZeldovich effect of discrete Ly $\alpha$ absorption systems. This result is based on the observed statistical properties of the Ly $\alpha$ forest in the spectra of QSOs (cf. eq. [8]). The predicted anisotropy signal appears on angular scales $\sim 0 ! 1-1^{\prime}$ and decreases roughly as $\propto \theta^{-1}$ on larger scales. Existing VLA observations place an upper limit of $(\Delta T / T)<2 \times 10^{-5}$ on an angular scale of 1.3 (Fomalont et al. 1993), which is consistent with the prediction of equation (9). The predicted effect dominates over the anisotropies produced by Bremsstrahlung emission from the same Ly $\alpha$ absorption systems (Loeb 1996) for wavelengths $\lambda \lesssim 3 \mathrm{~cm}$.

The magnitude of the expected signal from the Ly $\alpha$ forest is comparable to the Ostriker-Vishniac effect, which evaluates the anisotropies due to bulk velocities during early reionization using second-order perturbation theory (Ostriker \& Vishniac 1986; Vishniac 1987; Hu \& White 1995). However, it is important to emphasize that the predictions of this Letter rely on the observed population of $\operatorname{Ly} \alpha$ absorption systems with a minimal level of speculation about the full reionization history of the universe. While the thermal SZ effect from clusters of galaxies may also add fluctuations of comparable magnitude on the same angular scale (Colafrancesco et al. 1994, 1995), it is possible to separate it from the kinematic SZ effect of Ly $\alpha$ systems either by spectral measurements in several microwave bands or by a cross-correlation analysis with the fluctuations of the X-ray sky. The dominant contamination to the signal from Ly $\alpha$ systems comes from radio sources (see Figs. 9-11 in Tegmark \& Efstathiou 1996). Although their contribution to the anisotropies on arcminute scales is large, radio sources could, in principle, be removed through high-resolution multiple-frequency observations (Fomalont et al. 1993; Windhorst et al. 1993).

If the anisotropies caused by $\operatorname{Ly} \alpha$ absorption systems are detected, they would provide valuable information about the 
cosmic velocity field and the gas content of these systems at high redshifts. The two-dimensional information imprinted on the microwave sky could, in principle, be extended into redshift space, through a cross-correlation analysis with absorption spectra of QSOs in the same region of the sky. When extended surveys of QSOs, such as the forthcoming Sloan Digital Sky Survey (Gunn \& Weinberg 1995), become avail- able, it will be possible to perform such a correlation study over large areas of the sky.

I thank Daniel Eisenstein, Tsafrir Kolatt, and Uros Seljak for useful discussions. This work was supported in part by the NASA ATP grant NAG5-3085.
Bajtlik, S., Duncan, R. C., \& Ostriker, J. P. 1988, ApJ, 327, 570

Bechtold, J. 1994, ApJS, 91, 1

Bechtold, J., Crotts, A. P. S., Duncan, R. C., \& Fang, Y. 1994, ApJ, 437, L83

Birkinshaw, M. 1993, in Lecture Notes in Physics 429, Present and Future of the Cosmic Microwave Background, ed. J. L. Sanz, E. Martinéz-Gonzáles, \& L. Cayón (Berlin: Springer), 6

Blades, J. C., Turnshek, D. A., \& Norman, C. A. 1988, QSO Absorption Lines: Probing the Universe (Cambridge: Cambridge Univ. Press)

Carswell, R. F., Morton, D. C., Smith, M. G., Stockton, A. N., Turnshek, D. A. \& Weymann, R. J. 1984, ApJ, 278, 486

Cen, R., Miralda-Escudé, J., Ostriker, J. P., \& Rauch, M. 1994, ApJ, 437, L9 Colafrancesco, S., Mazzotta, P., Rephaeli, Y., \& Vittorio, N. 1994, ApJ, 433 454

454. 1995, in Ann. NY Acad. Sci., 759, 17th Texas Symp. on Relativistic Astrophysics and Cosmology, ed. H. Böhringer, G. E. Morfill, \& J. E. Trümper, 722

Dinshaw, N., Foltz, C. B., Impey, C. D., Weymann, R. J., \& Morris, S. L. 1995, Nature, 373, 223

Dinshaw, N., Impey, C. D., Foltz, C. B., Weymann, R. J., \& Chaffee, F. H. 1994, ApJ, 437, L87

Fang, Y., Duncan, R. C., Crotts, A. P. S., \& Bechtold, J. 1995, preprint (astro-ph/9510112)

Fixsen, D. J., Cheng, E. S., Gales, J. M., Mather, J. C., Shafer, R. A., \& Wright, E. L. 1996, ApJ, in press (COBE preprint no. 96-11)

Fomalont, E. B., Partridge, R. B., Lowenthal, J. D., \& Windhorst, R. A. 1993 ApJ, 404, 8

Górski, K., Davis, M., Strauss, M. A., White, S. D. M., \& Yahil, A. 1989, ApJ, 344,1

Gunn, J. E., \& Weinberg, D. H. 1995, in Wide Field Spectroscopy and the Distant Universe, ed. S. J. Maddox \& A. Aragón-Salamanca (Singapore: World Scientific), in press

Haehnelt, M. G., \& Tegmark, M. 1995, MNRAS, submitted (preprint astro-ph/9507077)

Hernquist, L., Katz, N., Weinberg, D. H., \& Miralda-Escudé, J. 1996, ApJ, 457, L51

Hu, W., \& White, M. 1995, A\&A, submitted (preprint astro-ph/9507060)

Janssen, M. A., et al. 1996, ApJ, submitted (preprint astro-ph/9602009)

Lanzetta, K. M., Wolfe, A. M., \& Turnshek, D. A. 1995, ApJ, 440, 435

\section{RFERENCES}

Levin, J. J., Freese, K., \& Spergel, D. N. 1992, ApJ, 389, 464

Loeb, A. 1996, ApJ, 459, L5

Lu, L., Wolfe, A. M., \& Turnshek, D. A. 1991, ApJ, 367, 19

Miralda-Escudé, J., Cen, R., Ostriker, J. P., \& Rauch, M. 1996, ApJ, in press (preprint astro-ph/9511013)

Ostriker, J. P., \& Vishniac, E. T. 1986, ApJ, 306, L51

Peebles, P. J. E. 1993, Principles of Physical Cosmology (Princeton: Princeton Univ. Press)

Persi, F. M., Spergel, D. N., Cen, R., \& Ostriker, J. P. 1995, ApJ, 442, 1

Pettini, M., Lipman, K., \& Hunstead, R. W. 1995, ApJ, 451, 100

Rauch, M., \& Haehnelt, M. G. 1995, MNRAS, 275, L76

Rephaeli, Y. 1995, ARA\&A, 33, 541

Sargent, W. L. W., Steidel, C. C., \& Boksenberg, A. 1989, ApJS, 69, 703

Sargent, W. L. W., Young, P. J., Boksenberg, A., \& Tytler, D. 1980, ApJS, 42, 41

Sembach, K. R., Steidel, C. C., Macke, R. J., \& Meyer, D. M. 1995, ApJ, 445, L27

Smette, A., Robertson, J. G., Shaver, P. A., Reimers, D., Wisotzki, L., \& Köhler, Th. 1995, A\&AS, 113, 199

Spitzer, L. 1978, Physical Processes in the Interstellar Medium (New York: Wiley)

Stengler-Larrea, E. A., et al. 1995, ApJ, 444, 64

Storrie-Lombardi, L. J., McMahon, R. G., Irwin, M. J., \& Hazard, C. 1994, ApJ, L13

Strauss, M., Cen, R., \& Ostriker, J. P. 1993, 408, 389

Strauss, M. A., \& Willick, J. A. 1995, Phys. Rep., 261, 271

Sunyaev, R. A., \& Zeldovich, Ya. B. 1980, ARA\&A, 18, 537

Tegmark, M., \& Efstathiou, G. 1996, MNRAS, 281, 1297

Tytler, D. 1987, ApJ, 321, 49

Vishniac, E. T. 1987, ApJ, 322, 597

Walker, T. P., Steigman, G., Kang, H., Schramm, D. N., \& Olive, K. A. 1991, ApJ, 376, 51

Webb, J. K., \& Barcons, X. 1991, MNRAS, 250, 270

Windhorst, R. A., Fomalont, E. B., Partridge, R. B., \& Lowenthal, J. D. 1993, ApJ, 405, 498

Wright, E. L., et al. 1994, ApJ, 420, 450

Zhang, Y., Anninos, P., \& Norman, M. L. 1995, ApJ, 453, L57 